\title{
Emerging Data in Gut Microbiome
}

\begin{abstract}
Aktay AN*
Department of Pediatric Gastroenterology, Hepatology and Nutrition, Rainbow Babies and Children's Hospital, Cleveland, USA

*Corresponding author: Atiye Nur Aktay, Department of Pediatric Gastroenterology, Hepatology and Nutrition, Rainbow Babies and Children's Hospital, Cleveland, USA
\end{abstract}

Received: April 18, 2017; Accepted: May 12, 2017;

Published: May 19, 2017

\section{Short Communication}

The research about gut microbiome has been growing at a very fast speed in the last 10 years. Since the Human Microbiome Project (HMP) was launched in 2007, our understanding of the role and function of the gut microbiota have changed dramatically. Human Genome project successfully identified that humans are made up of only 23,000 genes, only 400 genes different than chimpanzee and surprisingly $95 \%$ similar to mice. We thought humans were the most sophisticated, definitely more than animals and plants but even plants have more genes than we do. We now understand that the missing link is the human microbiome is the gut microbiome. The use of newer DNA sequencing methods helped the researchers to study the gut microbiome without culturing the samples in the laboratory. This approach is called "Metagenomics" allows the analysis of genetic material harvested directly from microbial communities. Other technologies like transcriptomics, proteomic and metabolomics measure the biologic properties of the microbiome. The HMP Consortium has reported the structure and function of the human microbiome at 18 body sites in 300 healthy adults (18-40 years old). The results have shown that microbial cells outnumber the human cells about 10:1 and have more microbial genes $(1,000,000+)$ than the human genes $(23,000)$ in our body.

Our gut microbiome controls our body with the help of immune and neuron cells and serves as the gatekeeper. If the conditions and balance is right then the factory works very well, but it microbiome is unbalanced that it sets off the cascade of events that leads to inflammation and chronic autoimmune conditions. How the balance of this gut ecosystem is is maintained? How do we acquire microbes in our gut? Well studies so far shows that newborn gets the first micrbiome from the vagina during birth then the number of microbiome increases until 3 years of age. Any disruption during the first 1000 days, such as antibiotic use, infections, westernized diet triggers inflammation either that time or later in life. This sophisticated well-orchestrated system requires bidirectional communication between microbiome and the host.
We just started understanding the importance of the gut microbiome and its symbiotic relationship with the host. The data regarding the role of gut microbiomes in Obesity, Non-alcoholic steatohepatitis (NASH), Inflammatory Bowel Disease (IBD), neuropsychiatric disorders and leaky gut are mounting. Recent studies in mice have shown that obese mice lose weight when transplanted with the microbiota of the lean mice. In Netherlands, researchers have found that the insulin sensitivity in male patients improved after the transplantation of lean donor's microbiota. Sonnenburg research in mice has shown that fiber rich diet increases the diversity of gut microbiota. Basically fiber is fermented by the gut bacteria into short chain fatty acids, which is important to maintain the gut barrier. Increased permeability allows translocation of bacteria and endotoxins into the blood stream, which results in low-grade inflammation and exaggerated immune response. This may lead to metabolic syndrome, NASH and Inflammatory bowel disease. Fecal microbial transplantation has been proven to treat resistant Clostridium Difficile infections. The second phase of the HMP focuses on the role of microbiota in 3 main disorders: onset of type 1 diabetes, onset of IBD and preterm labor-pregnancy.

It will take time to gather more data from these studies so what can we do in the meantime to optimize the gut health in children? I think it is important to main the equilibrium of microbiota in the gastrointestinal tract. Now, we know that gut microbes begins to develop during birthing process and resemble to the composition of adult gut microbiota (Firmicutes and Bacteriodetes dominating) by the age of 3 . However, this equilibrium is often disrupted by antibiotic use and westernized diet such as greasy fatty foods, meat and low fiber. Fermented products especially kefir and yogurt has the highest content of bacteria such as Lactobacillus, and Bifidobacterum. Pediatricians should be conscientious about the use of antibiotics, which often sweeps away billions of gut microbes. Unfortunately probiotics may not replace the lost microbes.

Given all of this information, we should educate pediatrician, parents, and children regarding the healthier diet (more vegetables, Mediterranean diet, fish, high fiber), fermented products such as Kefir, yogurt, Kimchi and avoiding the antibiotics as much as possible. Main problem in westernized diet is processed foods, which might be setting the gut ecosystem to dysbiosis. 